\title{
Influence de la finesse de broyage des aliments sur l'importance des pertes en particules dans la technique in sacco
}

\author{
B Michalet-Doreau, P Cerneau \\ CRZV station de recherche sur la nutrition des herbivores, \\ unité de la valeur alimentaire, \\ Theix, 63122 St-Genès-Champanelle, France
}

\begin{abstract}
Summary - Effect of substrate particle size on particulate matter losses from the bags in the in sacco technique. In sacco dry matter (DM) and nitrogen (N) losses after washing with a buffer were measured in 6 feeds ( 2 hays and 4 concentrates) with different fineness of grinding. Only for pea, barley and maize did finer grinding increase nitrogen losses. Moreover, these losses of nitrogen were lower than the nitrogen present in particles smaller than $50 \mu \mathrm{m}$.
\end{abstract}

Dans la méthode in sacco, une fraction non négligeable de particules alimentaires peut passer à travers les pores du sachet et être à l'origine d'une surestimation de la dégradabilité de l'aliment (Michalet-Doreau, 1990). Cette quantité de matière sèche perdue est fonction de la taille des pores du sachet (Lindberg et Knutsson, 1981), mais aussi de la finesse de broyage de l'aliment initial. Or, pour une même grille de broyage, la granulométrie de l'échantillon varie avec la nature de l'aliment. Dans cet essai, nous avons cherché d'une part, à mesurer les variations de pertes en particules en fonction de la finesse de broyage de l'échantillon et d'autre part, à relier ces pertes à la granulométrie de l'échantillon.

Matériel et méthodes - Cinq aliments (1 foin de graminée, 1 foin de légumineuse, 1 orge, 1 maïs, 1 pois) ont été broyés, sans séchage préalable, à la grille de $0,8,3$ ou $6 \mathrm{~mm}$, et un tourteau de soja à la grille de 0,8 et de $3 \mathrm{~mm}$. Des sachets (dimensions internes: $6 \times 11 \mathrm{~cm}$; taille des pores: $46 \mu \mathrm{m}$ ) contenant $3 \mathrm{~g}$ d'aliment broyé ont été placés dans un tampon borate-phosphate à pH 8 (1 sachet dans $250 \mathrm{ml}$ de tampon) et agités pendant $2 \mathrm{~h}$ dans un bain-marie à agitation (90 oscillations par min). Les pertes en particules ont été obtenues en filtrant cette solution. Par ailleurs, la granulométrie des aliments a été déterminée par tamisage en milieu humide (Grenet, 1984), sur 8 tamis dont l'ouverture de maille était comprise entre $4 \mathrm{~mm}$ et $50 \mu \mathrm{m}$. L'eau de rinçage contenant les particules les plus fines $(<50 \mu \mathrm{m})$ a été filtrée. La taille des particules des échantillons est caractérisée par sa moyenne arithmétique (Israelsen, 1968). La détermination des pertes en particules est effectuée 2 fois et celle de la granulométrie des aliments 3 fois.

L'azote des aliments et des particules retenues sur les filtres a été déterminée par la méthode Kjeldahl.

Résultats et discussion - La taille moyenne des particules augmente significativement avec le diamètre de la grille de broyage quel que soit l'aliment 
considéré. Elle passe de $0,5 \mathrm{~mm}$ à $2,8 \mathrm{~mm}$ pour le foin de graminée broyé à $0,8 \mathrm{~mm}$ ou à $6 \mathrm{~mm}$, et de 0,2 à $1,0 \mathrm{~mm}$ pour le pois. Parallèlement, le pourcentage de matière sèche (MS) perdue à travers les pores du sachet diminue significativement avec la diminution de la finesse de broyage, soit pour le maïs $17,9 \%$ avec le broyage à $0,8 \mathrm{~mm}$ contre $13,1 \%$ à $6 \mathrm{~mm}, 31,2$ contre $19,8 \%$ avec l'orge et 50,8 contre $29,4 \%$ avec le pois. II en est de même pour le pourcentage d'azote perdu, bien que la fraction azotée soit nettement plus faible que la fraction MS, soit $14 \% \mathrm{~N}$ perdu avec le pois broyé à $0,8 \mathrm{~mm}$ contre $50,8 \%$ de MS. Les pertes en particules ne varient pas de façon significative avec la finesse de broyage pour les 2 foins et le tourteau.

Par ailleurs, si la méthode utilisée pour mesurer les pertes reflète correctement les conditions d'incubation dans le rumen, la fraction de très fines particules $(50 \mu \mathrm{m})$ correspond à la fraction perdue à travers les pores du sachet $(46 \mu \mathrm{m})$. Or, le pourcentage de pertes en particules est étroitement lié à la quantité de très fines particules (fig 1), bien qu'il soit en moyenne inférieur à cette fraction de très fines particules. Les particules dont la taille est suffisamment petite pour passer à travers les pores seraient donc en partie retenues dans le sachet par suite soit d'un effet de colmatage des pores du tissu (Lindberg, 1981), soit de l'emprisonnement de ces particules dans un réseau de particules de taille plus importante.

Grenet E (1984) In: Techniques in particle size analysis of feed and digesta in ruminants (Kennedy PM, ed) Canadian Society of Animal Science, Edmonton, 167-168

Israelsen M (1968) Bioteknisk Inst Rep

Lindberg JE (1981) Swed J Agric Res 11, 7176

Lindberg JE, Knutsson PG (1981) Agric Environ $6,171-182$

Michalet-Doreau B (1990) Reprod Nutr Dev (suppl 2), 151s-152s

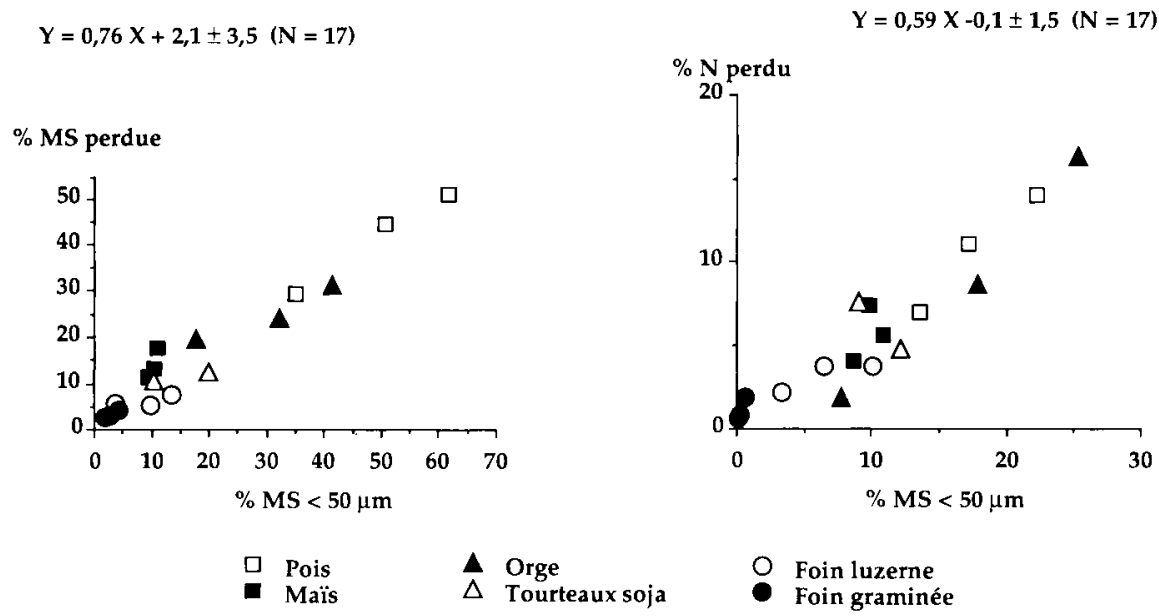

Fig 1. Liaison entre les pertes en particules (en $\%$ MS ou $N$ ) et le pourcentage de MS ou d'N alimentaire se trouvant dans les particules $<50 \mu \mathrm{m}$. 